\title{
Quantum-Based Photonic Sensors for Pressure, Vacuum, and Temperature Measurements: A Vison of the Future with NIST on a Chip
}

\author{
J Hendricks, Z Ahmed, D Barker, K Douglass, S Eckel, J Fedchak, N Klimov, J Ricker, J Scherschligt \\ National Institute of Standards and Technology, Physical Measurement Laboratory, 1 Sensor Science \\ Division, Thermodynamic Metrology Group, 100 Bureau Drive, Gaithersburg, MD 20899-8363 \\ E-mail: jayh@nist.gov
}

\begin{abstract}
Summary:
The NIST on a Chip (NOAC) program's central idea is the idea that measurement technology can be developed to enable metrology to be performed "outside the National Metrology Institute" by the creation of deployed and often miniaturized standards. These standards, when based on fundamental properties of nature, are directly tracible to the international system of units known as the SI. NIST is also developing quantum-based standards for SI traceability known as QSI, or Quantum based International System of units. Specifically, this paper will cover NIST efforts in the area of thermodynamic metrology to develop NOAC standards for pressure, vacuum and temperature measurements.
\end{abstract}

\section{Keywords:}

\section{Introduction}

For pressure measurements, this paper will cover NIST efforts to eliminate mercury manometers with a photonic based measurement based on ultra-precise measurements of gas refractive index. For vacuum measurements, this paper covers NIST efforts to develop a new a vacuum standard for measuring and understanding the pascal at the lowest pressures through the development of a cold atom vacuum standard. For temperature measurements, this paper covers NIST efforts to develop a method of measuring temperature using a photonic-based method.

The unifying theme is that all these efforts are aimed at the development of standards and sensors that are small, deployable, and based on fundamental physics, or are quantum-based. This has been embodied within the "NIST on a Chip" or NOAC program. The core the idea of NOAC is that quantum-based measurements are fundamental and when employed in sensors will not require re-calibration. In this embodiment, the standards lab, or in this case "NIST", is "on a chip" and is powerful to industry and society as it means that large networks sensors (or sensors "integrated" into a product or device) can be deployed and trusted to pro- vide accurate measurements without costly recalibration.

\section{Pressure}

Moving forward, the next generation of pressure standards will provide a new route of SI traceability for the pascal. By taking advantage of both the properties of light interacting with a gas and that the pressure dependent refractive index of helium can be precisely predicted from fundamental, first-principles quantum-chemistry calculations, a new route of realizing the pascal has been demonstrated.

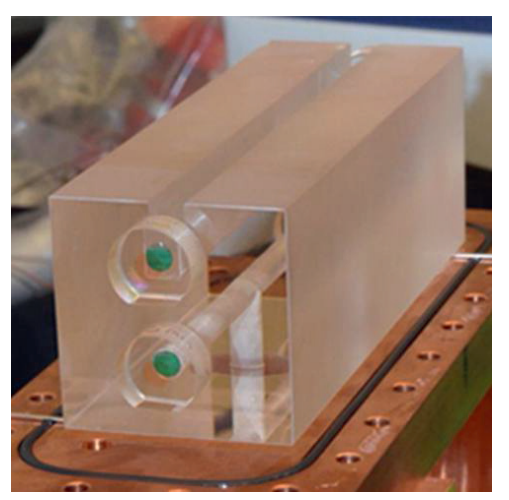

Figure 1: Fixed Length Optical Cavity (FLOC) will replace all mercury Manometers 
This technique is very different from classical methods of realizing pressure that have served the metrology community well for the past 375 years. The new photonic-based pressure standards will enable the elimination of mercury manometers, replacing them with a smaller, lighter, faster, and higher precision standard. From a metrology standpoint, the new quantum-based SI pascal will move us from the classical force/area definition, to an energy density (joules per unit volume) definition. Should the technique be further miniaturized, it will lead to a revolution in pressure metrology, enabling a photonics-based device that serves both a gas pressure sensor and a portable gas pressure standard all in one. In the future, the mercury barometer will be replaced with a new standard based on quantum chemistry calculations.

The new method relies on a pair of optical cavities, each consisting of a set of mirrors on a spacer with the gas/vacuum filling the space between the mirrors. To improve upon this design, the reference cavity is always kept at vacuum to help eliminate noise and other systematic errors. This device, referred to as a Fixed Length Optical Cavity (FLOC), is shown in Figure 1. The FLOC is made out of a glass with Ultra-Low thermal Expansion (ULE) to prevent changes in interferometer length with temperature. The upper cavity consists of a slot to allow gas to easily flow in and out, whereas the reference cavity is a hole drilled through the glass block and sealed at either end via mirrors. [1-4]

Additionally, a vertical tube allows one to pump out the reference cavity through a vacuum pump. The glass cavity is placed inside a chamber to improve temperature stability and to ensure that the gas species is known, and therefore has a known refractivity. For gasses such as helium with simple electron structure and limited number of isotopes, the refractivity and density virial coefficients can be calculated through quantum mechanics [2]. This calculation can provide refractivity to an uncertainty better than 1 parts in 106 . For other gasses, the values must be measured or will be calculated but with significantly larger uncertainties. Because the calculation of pressure is only dependant on refractivity and temperature, we can define the FLOC as a primary realization of pressure.

The FLOC is primary but does require corrections be carefully accounted for when making a high accuracy pressure measurement with the lowest possible uncertainties. The first correction is the distortion of the glass when pressure is applied. The glass experiences a bulk com- pression when forces to the outside surfaces occur. In addition to the bulk compression, the glass experiences a non-uniform bending due to the reference cavity being at a different pressure. While these distortion corrections are different for each glass cavity, the value can be determined experimentally and corrected with high accuracy. The second correction that must be accounted for is that helium can absorb into glass causing the glass to change its dimensions. By collection interferometer data the absorption can be traced over time and extrapolated back to zero, with high accuracy.

Overall a FLOC standard can achieve an uncertainty of 9 parts in 106 in nitrogen [4]. It is anticipated that a better determination of this index will soon allow this to be drastically reduced. Additionally, the best method to measure pressure distortions is to use several gasses of known refractive index at the same pressure. The distortions will be independent of gas species and can be solved to determine the magnitude of the error and even lower uncertainties. This means that as lower and lower uncertainty refractivity measurements are made by independent labs, the lower the uncertainty the FLOC method becomes world-wide. This is the power of quantum-based measurements.

\section{Vacuum}

For vacuum measurements, NIST efforts to develop a new vacuum standard for measuring and understanding the pascal at the lowest pressures is underway. To achieve this, the Cold- Atom Vacuum Standard (CAVS) has been developed which uses a cold atom trap to sense pressure. [5] Since the earliest days of neutral atom trapping, it has been known that the background gas in the vacuum limits the trap lifetime (the characteristic time that atoms remain trapped). NIST is inverting this problem to create a quantum-based standard and sensor.

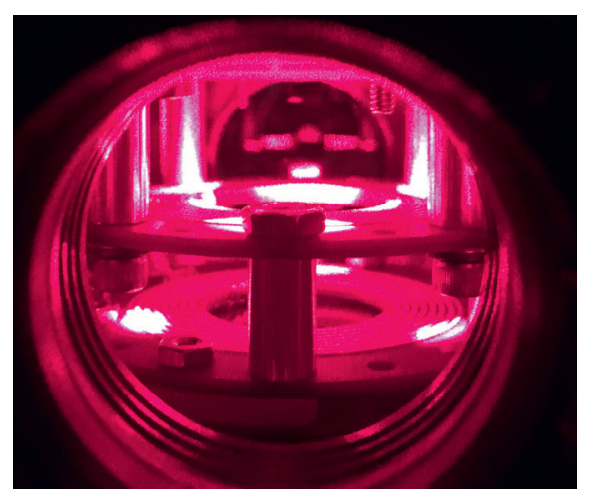

Figure 2: CAVS small portable version NIST prototype with a cloud of trapped $\mathrm{Li}$ atoms. 
Because the measured loss-rate of ultra- cold atoms from the trap depends on a fundamental atomic property (the loss-rate coefficient, related to the thermalized cross section) such atoms can be used as an absolute sensor and primary vacuum standard. Researchers have often observed that the relationship between the trap lifetime and background gas can be an indication of the vacuum level, and several research groups have pursued using cold atom traps as vacuum sensors. [6,7] However, an absolute vacuum standard, sufficient for use as an international quality standard, has not yet been realized. To do this requires rigorous attention to all potential error sources, from both the atomic perspective and the vacuum perspective. Moreover, a primary CAVS requires the collision cross section between trapped ultra-cold atoms and the background gas to be traceable to an ab initio theoretical determination. NIST has built a laboratory-scale CAVS apparatus, developed the measurement scheme, and done preliminary theoretical calculations, all of which show promising early results. In addition, NIST is developing a small, portable version that uses a grating-based trap (shown in Figure 2) that will eventually enable users to realize and measure vacuum pressures in their lab without relying on calibrated sensor artifacts.

\section{Temperature}

For temperature measurements, NIST efforts to develop a method of measuring temperature using a photonic-based method are underway. Temperature measurements and sensors play a crucial role in various aspects of modern technology ranging from medicine and manufacturing process control, to environmental and oil-and-gas industry. Among various temperature measurement solutions, resistance-based thermometry is a time-tested method of disseminating temperature standards. [9]

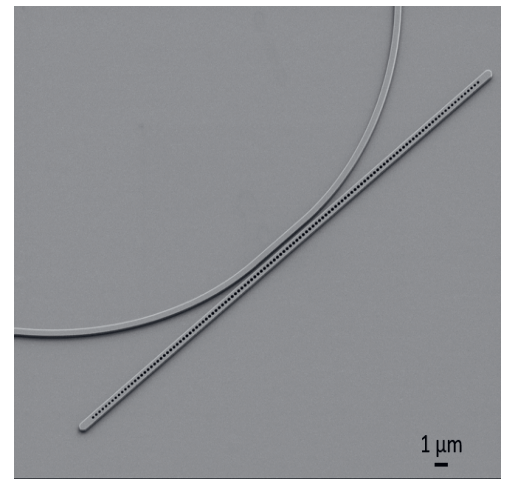

Figure 3. Silicon photonic crystal cavitythermometer fabricated at NIST
Although industrial resistance thermometers can routinely measure temperatures with uncertainties of $10 \mathrm{mK}$, their performance is sensitive to multiple environmental variables such as mechanical shock, thermal stress and humidity. These fundamental limitations of resistance thermometry, as well as the desire to reduce sensor ownership cost, have ignited a substantial interest in the development of alternative temperature measurement solutions such as photonics-based temperature sensors $[10,11]$. NIST is developing novel on-chip integrated silicon photonic temperature sensors with nanoscale footprint and ultra-high resolution as an alternative solution to legacy-based resistance thermometers. These sensors are Fabry-Perrot cavity type silicon photonic devices that are based on a Photonic Crystal nanobeam Cavity (PhCC), whose high-Q resonant frequency mode is highly sensitive to even ultra-small temperature variations. NIST has performed the first direct comparison of our photonic thermometers to Standard Platinum Resistance Thermometers, the best in class resistance temperature sensors used to disseminate the International Temperature Scale of 1990. The preliminary results indicate that our PhCC nanothermometers can detect changes of temperature as small as sub-10 $\mu \mathrm{K}$ and can achieve measurement capabilities that are on-par or even better than the state-of-the-art resistance thermometry.

\section{References}

[1] P. Egan, J. Stone, J. Hendricks, J. Ricker, G. Scace, G. Strouse, "Performance of a dual Fabry-Perot cavity refractometer," Opt. Letters, Vol. 40, No. 17, August 2015

[2] M. Puchalski, K. Piszczatowski, J. Komasa, B. Jeziorski, K. Szalewicz, 2016, "Theoretical determination of the polarizability dispersion and the refractive index of helium," Amer. Phys. Soc., Phys. Rev. A

[3] P. Egan, J. Stone, J. Ricker, J. Hendricks, 2016, "Metrology for comparison of displacements at the picometer level," Amer. Inst. of Phys., Rev. of Sci. Inst. 87, 053113

[4] J. Stone, P. Egan. J. Hendricks, G. Strouse, D. Olson, J. Ricker, G. Scace, D. Gerty, 2015 "Metrology for comparison of displacements at the picometer level," Key Eng. Mat. Vol. 625 p 79-84

[5] J. Scherschligt, J. A. Fedchak, D.S. Barker, S. Eckel, N. Klimov, C. Makrides, and E. Tiesinga, Metrologia 54, S125 (2017).

[6] D.E. Fagnan, J. Wang, C. Zhu, P. Djuricanin, B.G. Klappauf, J.L. Booth, and K.W. Madison, Phys. Rev. A - At. Mol. Opt. Phys. 80, 1 (2009). 
[7] T. Arpornthip, C.A. Sackett, and K.J. Hughes, Phys. Rev. A - At. Mol. Opt. Phys. 85, 1 (2012).

[8] S. Eckel, D. Barker, J. Fedchak, N. Klimov, E. Norrgard and J. Scherschligt, Metrologia (submitted 2018).

[9] Strouse, NIST Spec. Publ. 250, 81 (2008).

[10] Kim et al., Opt. Express 18, 22215 (2010).

[11] Proceedings Volume 10923, Silicon Photonics XIV; 109230L (2019) https://doi.org/10.1117/12.2505898 Event: SPIE OPTO, 2019, San Francisco, California, United States

[12] Douglass, K.O. and D.A. Olson 2016 Towards a standard for the dynamic measurement of pressure based on laser absorption spectroscopy. Metrologia. 53(3): p. S96-S106.

[13] Ahmed, Z., D. Olson, and K.O. Douglass 2016 Precision Spectroscopy to Enable Traceable Dynamic Measurements of Pressure. CLEO: $p$. ATu1J.1.

[14] Hanson, E., et al. 2018 Towards traceable transient pressure metrology. Metrologia. 